\title{
Challenges faced by primary care services regarding access to care and mental health - the reality of a family health unit in Portugal during the Covid-19 Pandemic
}

\author{
Hannah Gough* and Joana Paço \\ Family medicine resident at USF Andreas, Largo Coronel Brito Gorjão, Mafra, Portugal
}

\begin{abstract}
Primary health care plays an important role in diagnosing and managing mental health disorders, with access to care being a fundamental determinant in this area. This has been highlighted over the past year with the Covid-19 Pandemic. We have some observations to make regarding access to primary care services and prevalence of mental health disorders in Portugal during the Covid-19 Pandemic. Access to public primary care services in Portugal has been compromised significantly since the beginning of the Covid-19 Pandemic for many reasons. Despite the difficulty in seeing a doctor we have observed an increase in the number of mental disorders diagnosed in January to August 2020 as compared to the same period in 2019. Our health care centre serves a population of 18,498 people and we observed a $13.38 \%$ increase in sleep disturbance (P06) and a 6.5\% increase in depressive disorder (P76). The prevalence of anxiety disorder (P74) rose $8.99 \%$ from 2019 to 2020 and somatisation disorder (P75) rose $26.74 \%$ as compared to the same period in 2019. Suicide/suicide attempts (P77) increased by $8.1 \%$. With primary care services being the main entry point into health systems it can be easily understood that if there is a disruption or difficulty in accessing these services, effective treatment and support can be severely delayed for a person suffering from a mental health disorder. These effects need to be considered when redistributing human resources, namely primary care physicians in the fight against Covid-19.
\end{abstract}

We would like to highlight the importance of primary health care regarding mental health in light of the current Covid-19 Pandemic. The diagnosis of mental health disorders is often made in the primary health care setting, underlining the importance of this sector in light of the current global health situation. Early diagnosis is imperative to ensure timely treatment and to avoid long-term complications, however this presupposes easy access to health services.

We have some observations to make regarding access to primary care services and prevalence of mental health disorders during the Covid-19 Pandemic, based on our experience over the past year in Portugal.

Access to care during a pandemic can be jeopardised for many reasons. Socioeconomic inequalities in accessing a doctor may already exist, as is the case in most OECD countries, however inequalities are known to be largest in Portugal, Canada and the United States [1].

Portugal is the 5th EU country with the highest prevalence of mental health disorders (18.4\% of the population) [2]. Since the beginning of the Covid-19 Pandemic we have observed an increase in the prevalence of diagnosed mental health problems in a family health unit (USF Andreas) in Mafra, Portugal, despite access to primary health services being more difficult. Access to public primary care services in Portugal has been compromised significantly since the beginning of the Covid-19 Pandemic for many reasons. Many general practitioners who normally work in public health care centres across the country have been required to work in official Areas Dedicated to Covid-19 and Respiratory Diseases, making it impossible to maintain easy access for their patients. In addition to this, during their working hours in the health care centres, general practitioners have been overwhelmed with other bureaucratic tasks. This includes monitoring the health of
Covid-19 positive patients with mild symptoms in the form of a daily phone call, a task which has reached huge proportions over the last few months. Considering these factors and the fact that some physicians working in primary care have become ill themselves we can begin to understand how severely the access to care has been affected.

Despite the difficulty in seeing a doctor we have observed an increase in the number of mental disorders diagnosed in January to August 2020 as compared to the same period in 2019. Our health care centre serves a population of 18,498 people and we observed a $13.38 \%$ increase in sleep disturbance (P06) and a 6.5\% increase in depressive disorder (P76). The prevalence of anxiety disorder (P74) rose $8.99 \%$ from 2019 to 2020 and somatisation disorder (P75) rose $26.74 \%$ as compared to the same period in 2019. Suicide/suicide attempts (P77) increased by $8.1 \%$. Difficulty in accessing primary care is a nation-wide problem at the moment, most likely indicating a similar increase in mental health disorders across the country.

With primary care services being the main entry point into health systems it can be easily understood that if there is a disruption or difficulty in accessing these services, effective treatment and support can be severely delayed for a person suffering from a mental health disorder. These effects need to be considered when redistributing

${ }^{\star}$ Correspondence to: Hannah Gough, MD, Family medicine resident at USF Andreas, Largo Coronel Brito Gorjão, Mafra, 2640-465, Portugal, E-mail: hannah.gough.mail@gmail.com

Key words: access to care, covid-19, mental health

Received: January 05, 2021; Accepted: January 13, 2021; Published: January 17, 2021 
Gough H (2021) Challenges faced by primary care services regarding access to care and mental health - the reality of a family health unit in Portugal during the Covid-19 Pandemic

human resources, namely primary care physicians in the fight against Covid-19.

\section{Acknowledgment}

None.

\section{Funding}

None

\section{Conflicts of interest}

None.

\section{References}

1. OECD (2019) Health at a Glance 2019: OECD Indicators, OECD Publishing, Paris.

2. OECD (2020) Health at a Glance: Europe 2020: State of Health in the EU Cycle. OECD Publishing, Paris.

Copyright: $\odot 2021$ Gough H. This is an open-access article distributed under the terms of the Creative Commons Attribution License, which permits unrestricted use, distribution, and reproduction in any medium, provided the original author and source are credited. 